Provided for non-commercial research and education use. Not for reproduction, distribution or commercial use.

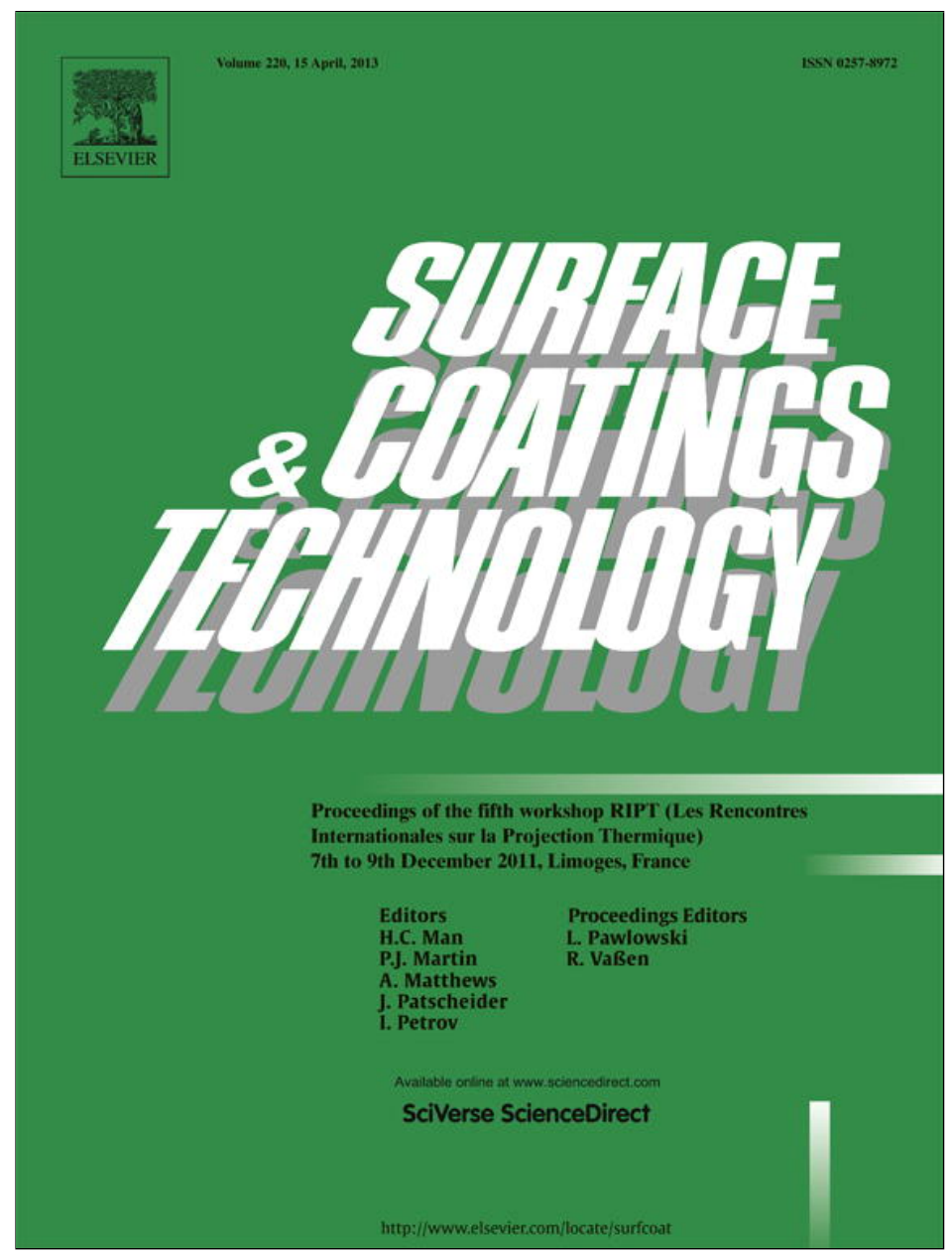

This article appeared in a journal published by Elsevier. The attached copy is furnished to the author for internal non-commercial research and education use, including for instruction at the authors institution and sharing with colleagues.

Other uses, including reproduction and distribution, or selling or licensing copies, or posting to personal, institutional or third party websites are prohibited.

In most cases authors are permitted to post their version of the article (e.g. in Word or Tex form) to their personal website or institutional repository. Authors requiring further information regarding Elsevier's archiving and manuscript policies are encouraged to visit:

http://www.elsevier.com/authorsrights 


\title{
Suspension plasma sprayed bioactive glass coatings: Effects of processing on microstructure, mechanical properties and in-vitro behaviour
}

\author{
A. Cattini ${ }^{\text {a,b,* }}$, L. Łatka ${ }^{\text {b,c }}$, D. Bellucci a , G. Bolelli a , A. Sola ${ }^{\text {a }}$, L. Lusvarghi ${ }^{\text {a }}$, L. Pawłowski ${ }^{\text {b }}$, V. Cannillo ${ }^{\text {a }}$ \\ a Department of Materials and Environmental Engineering, University of Modena and Reggio Emilia, 41125 Modena, Italy \\ b SPCTS, UMR CNRS 7315, University of Limoges, F-87068 Limoges, France \\ c Faculty of Mechanics, Wrocław University of Technology, 50-372 Wrocław, Poland
}

\section{A R T I C L E I N F O}

Available online 8 November 2012

\section{Keywords:}

Bioactive glass

Suspension plasma spray

Simulated body fluid

Scratch test

Depth-sensing indentation

\begin{abstract}
A B S T R A C T
Bioactive glass coatings deposited via suspension plasma spraying were studied to improve the adhesion between orthopaedic implants and bone. Fine powders of a bioactive glass, named BG_Ca, having composition (in wt.\%): $4.7 \mathrm{Na}_{2} \mathrm{O}, 42.3 \mathrm{CaO}, 6.1 \mathrm{P}_{2} \mathrm{O}_{5}$, and $46.9 \mathrm{SiO}_{2}$, were produced and dispersed in ethanol to form a suspension used as a feedstock. Various sets of spray parameters were applied in order to define the influence of the deposition process on the final coating properties. Consequently, the coatings were characterized in as-sprayed state and after soaking in a simulated body fluid (SBF) for different periods ranging from 1 to 14 days. The microstructural investigations were carried out using environmental scanning electron microscope (ESEM) and X-ray diffraction (XRD). The coatings' adhesion to the substrate was evaluated by means of scratch tests. Finally, hardness and elastic modulus were determined by means of depth-sensing indentation methods.
\end{abstract}

(c) 2012 Elsevier B.V. All rights reserved.

\section{Introduction}

Bioactive glasses are an emerging class of materials for biomedical applications [1]. Their increasing success depends primarily on their high bioactivity and flexibility, since the glass composition may be tailored to match the assigned biomedical requirements [2].

Silicate-based bioactive glasses usually belong to the $\mathrm{Na}_{2} \mathrm{O}-\mathrm{K}_{2} \mathrm{O}-\mathrm{CaO}-$ $\mathrm{MgO}-\mathrm{P}_{2} \mathrm{O}_{5}-\mathrm{SiO}_{2}$ system with appropriate proportions between the constituent oxides [3]. Their bone-bonding ability results from the prompt formation of a superficial layer of biomimetic hydroxy-carbonatedapatite (HCA) when exposed to a physiological environment [1]. Although the bioactivity of these systems makes them very useful for the production of prosthetic devices, their relatively poor mechanical properties including brittleness severely limit their use in loadbearing applications. In order to circumvent such restraints, bioactive glasses are preferentially applied as coatings onto tougher metallic substrates [4]. In this way, the substrate provides the required mechanical reliability, while the ceramic coating confers the desirable biocompatibility [5].

The plasma spray technique is currently the standard method to deposit bioactive glass coatings [6,7]. The present contribution introduces an innovative spray technique, namely the suspension plasma spray (SPS), in which a liquid suspension is used as feedstock instead of a dry powder. This techniques uses the sub-micrometric and nanometric powders what results in achievement of thinner coatings having finer

* Corresponding author at: 905, Via Vignolese, 41125 Modena, Italy. Tel.: + 39059 2056218, + 39059 2056233; fax: + 390592056243.

E-mail address: andrea.cattini@unimore.it (A. Cattini). and denser microstructure, in comparison with conventional plasmasprayed ones [8]. A bioactive glass, named BG_Ca [9-11], was chosen due to its low tendency to crystallise even at high temperature [10]. The various deposition parameters were tested to define the influence of deposition process conditions on final properties of coatings.

\section{Experimental methods}

\subsection{Suspension preparation}

The BG_Ca glass ( $4.7 \mathrm{Na}_{2} \mathrm{O}, 42.3 \mathrm{CaO}, 6.1 \mathrm{P}_{2} \mathrm{O}_{5}, 46.9 \mathrm{SiO}_{2}$ in oxide wt.\%) was produced by means of a standard melt-quenching method as described elsewhere $[9,10]$. The molten glass was splashed into roomtemperature water and the obtained frit was dried overnight at the temperature of $110{ }^{\circ} \mathrm{C}$. Then, the frit was milled in an agate jarand sieved through a $63 \mu \mathrm{m}$ sieve. The powders were further attrition milled in ethanol (95 vol.\%) using $0.8 \mathrm{~mm}$ zirconia balls with Beycostat C213 as a dispersant ( 2 wt.\% of the dry powder). Ethanol was chosen as milling and suspension medium since bioactive glasses react chemically with water [12]. The final powders had a monomodal distribution with a mean particle size of $4.9 \mu \mathrm{m}$ (Fig. 1), as determined with a Partica LA-950V2 (Horiba) granulometer. The feedstock suspension was formulated by dispersing 20 wt.\% of solid phase in 80 wt.\% of ethanol.

\subsection{Coating deposition}

In this study, a SG-100 torch (Praxair, S.T., Indianapolis, IN, USA), with an internal radial continuous-stream injector, was used. The injector was located inside the torch, $16 \mathrm{~mm}$ from its exit. During each spray run, the 


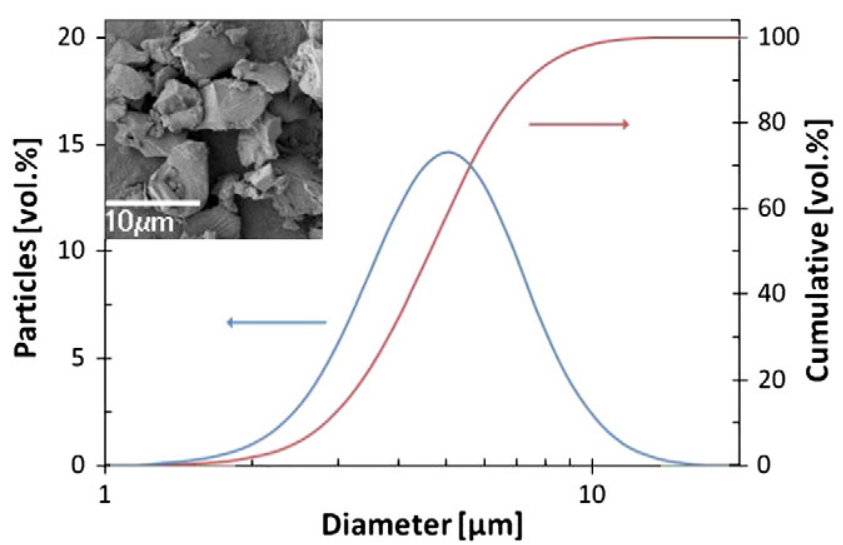

Fig. 1. Particle size distribution of feedstock powder.

suspension was continuously stirred in order to avoid any precipitation or aggregation phenomena.

In order to assess the feasibility of the new SPS technique, preliminary coatings were sprayed onto $316 \mathrm{~L}$ stainless steel discs (diameter: $25 \mathrm{~mm}$, thickness: $8 \mathrm{~mm}$ ) and the deposition parameters were changed over a very wide range, as specified in Table 1 , column 2 . Then, in order to refine the optimal parameters, five additional depositions were performed on Ti6Al4V titanium alloy discs (diameter: $25 \mathrm{~mm}$, thickness: $8 \mathrm{~mm}$ as before) according to the sets detailed in Table 1, column 3, and in Table 2. For clarity reasons, the coatings on Ti6Al4V were denoted as BGCa1-BGCa5 (Table 2). Since preliminary tests on mirror-like polished substrates showed that the coating adhesion was poor, the surface roughness was increased by sandblasting with $250 \mu \mathrm{m}$ corundum powder at a pressure of 5 bar to reach the roughness of $R_{\mathrm{a}}=3.4 \mu \mathrm{m}$. After sandblasting, the substrates were ultrasonically washed with ethanol and acetone.

During the final depositions on Ti6Al4V substrates, the coatings' surface temperature was monitored using a pyrometer IN 5 Plus (Impac). The mean value of the maximum temperature reached during the deposition is reported in Table 2.

\subsection{Mechanical property evaluation}

Scratch tests were performed on the as-sprayed coatings with the Open Platform Micro-Combi set-up (CSM Instruments, Switzerland)

Table 1

Optimization of spray parameters.

\begin{tabular}{lll}
\hline Spray parameters & $\begin{array}{l}\text { Screening of } \\
\text { parameters }\end{array}$ & $\begin{array}{l}\text { Final } \\
\text { depositions }\end{array}$ \\
\hline $\begin{array}{l}\text { Plasma spray } \\
\text { Substrate }\end{array}$ & 316 L stainless steel & Ti6Al4V \\
Electric power $[\mathrm{kW}]$ & 10 to 40 & See Table 2 \\
Working gas composition & Ar and $\mathrm{H}_{2}$ & \\
Argon flow rate $[\mathrm{slpm}]$ & 45 & 7.5 \\
Hydrogen flow rate $[\mathrm{slpm}]$ & 2.5 to 7.5 & See Table 2 \\
Spray distance $[\mathrm{mm}]$ & 40 to 80 & 4 \\
Torch scans for one session & 2 to 6 & 5 \\
Sessions for one experiment & 1 to 5 & 750 \\
Torch scan speed [mm/s] & 375 to 800 & 10 \\
Scan-step [mm] & 6 to 11 & \\
Cooling between sessions $\left[{ }^{\circ} \mathrm{C}\right]$ & Down to 70 & \\
& & 300 \\
Suspension injection & & 30 \\
Diameter of injector $[\mu \mathrm{m}]$ & 300 and 500 & $0.16-0.17$ \\
Suspension flow rate $[\mathrm{g} / \mathrm{min}]$ & From 20 to 60 & \\
Static pressure in suspension & $0.16-0.17$ & \\
$\quad$ container [MPa] & & \\
\hline
\end{tabular}

Table 2

Spray parameters

\begin{tabular}{llll}
\hline Experimental run & $\begin{array}{l}\text { Power } \\
{[\mathrm{kW}]}\end{array}$ & $\begin{array}{l}\text { Spray distance } \\
{[\mathrm{mm}]}\end{array}$ & $\begin{array}{l}\text { Maximum coating temperature, } \\
\text { mean value }\left[{ }^{\circ} \mathrm{C}\right]\end{array}$ \\
\hline BGCa1 & 36 & 50 & $344 \pm 34$ \\
BGCa2 & 40 & 50 & $569 \pm 36$ \\
BGCa3 & 38 & 60 & $473 \pm 29$ \\
BGCa4 & 36 & 70 & $278 \pm 32$ \\
BGCa5 & 40 & 70 & $461 \pm 47$ \\
\hline
\end{tabular}

equipped with a conical Rockwell-type diamond indenter with a spherical tip of $200 \mu \mathrm{m}$ radius. The applied load was increased linearly from $20 \mathrm{mN}$ to $30 \mathrm{~N}$, with a loading rate of $10 \mathrm{~N} / \mathrm{min}$ and a scratch length of $3 \mathrm{~mm}$. The acoustic emission was analysed in order to define the critical loads, whose value was also verified by ESEM-based optical analysis. For each sample, five scratch tests were carried out to obtain representative values.

Depth-sensing nano-indentation tests were performed on the coatings' cross-section. The samples were preliminary mounted in resin, cut and polished following a standard metallographic procedure. The maximum applied load was $50 \mathrm{mN}$, with a loading rate of $40 \mathrm{mN} / \mathrm{min}$ and a holding time of $15 \mathrm{~s}$. The elastic modulus was calculated according to the Oliver and Pharr method [13]. At least 15 indentations were performed for each sample. The mechanical characterization was focused on the samples BGCa1-BGCa5.

\subsection{In vitro tests}

In vitro tests were carried out on the BGCa1-BGCa5 samples by immersing them in a simulated body fluid (SBF) according to the standard protocol defined by Kokubo and Takadama [14]. The SBF volume-to-coating surface ratio was fixed to $20 \mathrm{~mL} / \mathrm{cm}^{2}$. The SBF was refreshed every 2 days in order to simulate the dynamic soaking conditions. The samples were extracted from the SBF, washed with bi-distilled water, dried at room temperature and characterized (morphology and chemical composition) after time periods of $1,3,7$, and 14 days.

\subsection{Microstructural analysis}

The surface and polished cross section of the coatings were observed with an environmental scanning electron microscope, ESEM (ESEM Quanta 200-FEI Company, Eindhoven, The Netherlands), operated in low-vacuum mode (pressure: 0.5 Torr) and equipped with a X-EDS microanalysis system (Inca, Oxford Instruments, U.K.). For selected samples, BGCa1-BGCa5, the ESEM evaluation was repeated after SBF tests. The coatings were also analysed by means of X-ray diffraction (XRD) before and after soaking in SBF, with a X'pert PRO diffractometer (PANalytical, Almelo, The Netherlands) employing a Cu-K $\alpha$ radiation. Data were collected in the $2 \theta$ range of $10^{\circ}-65^{\circ}$ with a step of $0.017^{\circ}$ and a time interval of $71.12 \mathrm{~s}$ (X'Celerator detector).

The surface of as-sprayed samples and SBF-soaked ones was investigated by micro-Raman Spectroscopy (Jobin-Yvon Raman Microscope Spectrometer, HORIBA Jobin Yvon S.A.S.,Villeneuve d'Ascq, France). He-Ne laser emitting at $632.8 \mathrm{~nm}$ was employed, with an output power of $20 \mathrm{~mW}$ without any filter and a $100 \times$ objective.

\section{Results and discussion}

\subsection{Screening of spray parameters}

A feasibility study was performed spraying more than 40 different coatings, as detailed in Table 1, column 2. The morphological and compositional evaluation of such exploratory samples via ESEM, XRD and micro- 
Raman spectroscopy revealed that the coatings' properties were governed mainly by following operational parameters:

- spray distance;

- electric power input to plasma;

- hydrogen content in working gas mixture.

A few initial spraying experiments were unsuccessful, since it was not even possible to melt the glass powder completely (Fig. 2). To overcome this problem, the electric power input was increased up to $40 \mathrm{~kW}$ and the hydrogen flow rate was augmented up to $7.5 \mathrm{slpm}$.

In this way, the glass particles reached the melting point, as confirmed by the ESEM observation. Indeed, due to the increase in the electric power input and the hydrogen flow rate, it is reasonable that the "Ability-of-Heating" factor (AHF) of the plasma [15,16], i.e. its capacity to heat and melt the particles, increases up to exceed the Difficultyof-Melting factor (DMF) of the powder (which depends on the heat content per unit mass of the particles, on the diameter of the largest particle in the distribution and on the density of the particle) $[15,16]$. In fact, the particle may be melted in plasma jet only if the parameter DMF which characterizes it is lower than the parameter AHF characterizing plasma jet [16]

The spray distance was another important parameter. In fact, the coatings contained the particles which re-solidified in-flight for spray distances greater than $70 \mathrm{~mm}$. On the contrary, the coating surface showed large drops of glass for spray distances smaller than $50 \mathrm{~mm}$ what proved that the convective heat flux from plasma jet melted the material.
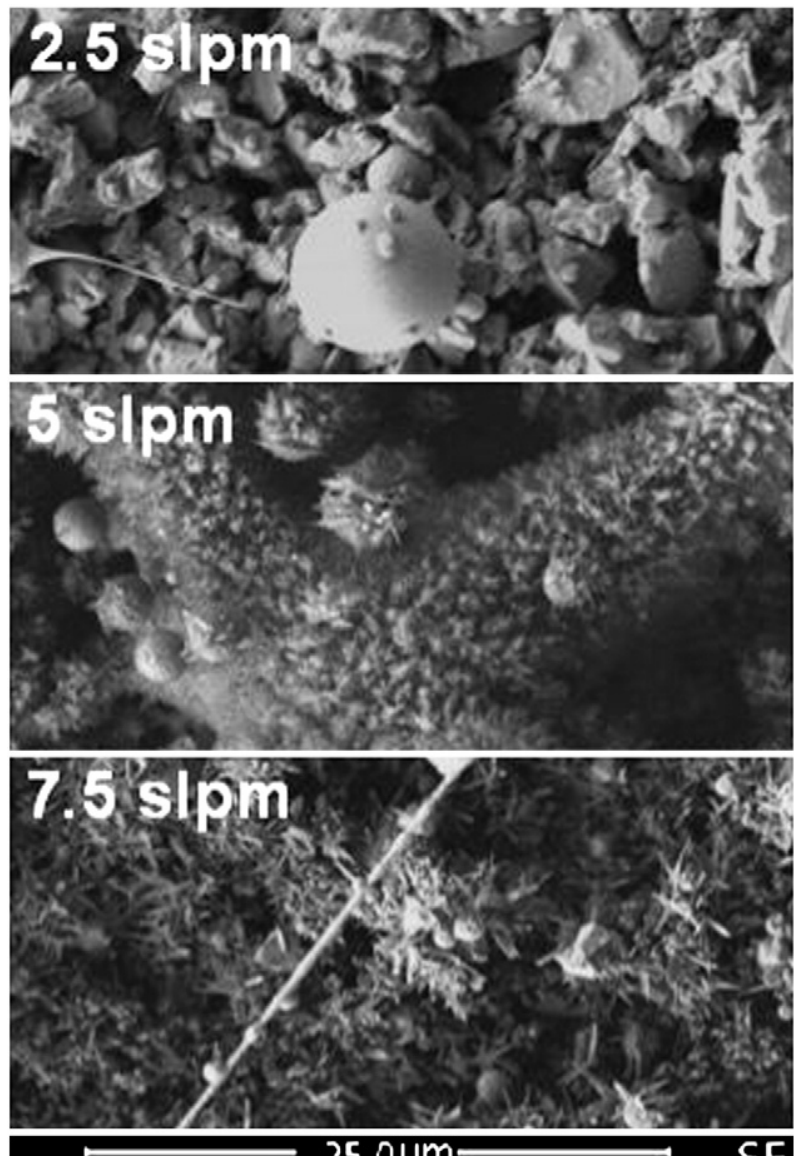

Fig. 2. Preliminary coatings produced with different hydrogen flow rates (2.5/5.0/7.5 slpm) The other spray parameters were set as follows: Ar flow rate $45 \mathrm{slpm}$; electric power input $25 \mathrm{~kW}$; spray distance $60 \mathrm{~mm}$; linear substrate speed $500 \mathrm{~mm} / \mathrm{s}$; scan-step $6 \mathrm{~mm}$; static suspension pressure $0.05 \mathrm{MPa}$; suspension feed rate $50 \mathrm{~g} / \mathrm{min}$.
The outcome of this initial parameters' screening for the depositions of BGCa1-BGCa5 samples was the following: (i) hydrogen flow rate was set to $7.5 \mathrm{slpm}$; (ii) electric power input was increased from 36 to $40 \mathrm{~kW}$; and, (iii) spray distance was fixed to be in the $50-70 \mathrm{~mm}$ range. Also, the injector, having an inner diameter (ID) of $300 \mu \mathrm{m}$, was preferred to the larger one of $500 \mu \mathrm{m}$. It appeared that the smaller ID of injector allowed a good penetration of the suspension into the plasma jet. The other parameters (scan speed, time of deposition, etc.) were selected in order to avoid an excessive heating of the substrate.

\subsection{Microstructure of as-sprayed final coatings}

The final coatings were continuous and homogeneous, regardless of the specific deposition parameters; in particular, the samples showed a very rough surface that included both large flattened splats and partially crystallised zones, as reported in Fig. 3a. A similar microstructure has been reported in the literature for hydroxyapatite coatings produced by SPS [17]. It is likely that such two-zone microstructure derives from the different thermo-mechanical behaviour of small and large particles in the feedstock powder, since they possess different heat capacities and follow dissimilar trajectories in the plasma jet [18]. Previous

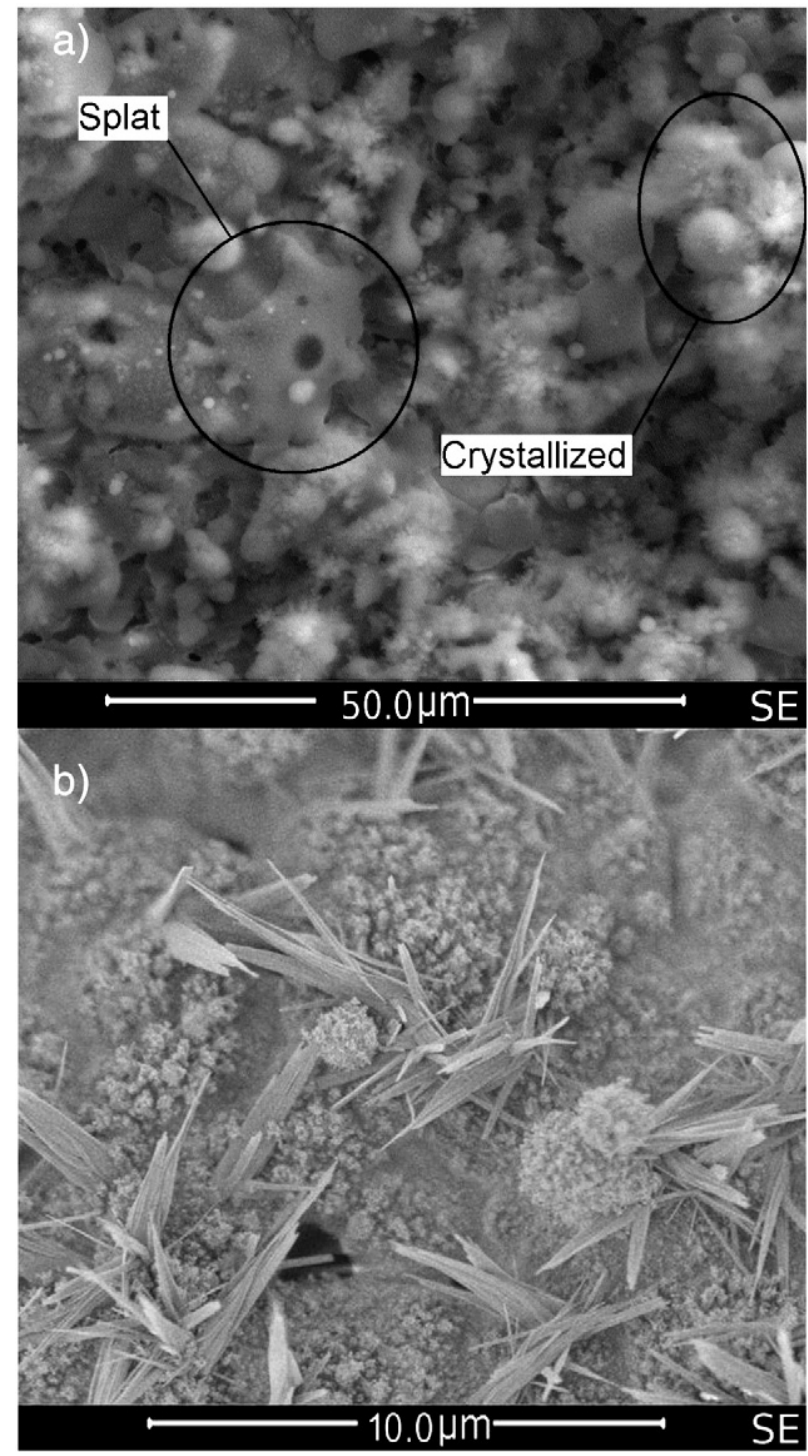

Fig. 3. SEM micrographs (secondary electrons): the area representative of the surface morphology of the coatings (BGCa3 run) with molten splats and crystallised zones outlined (a); high magnification of a crystallised area (b) (note that the acicular morphology typical of $\mathrm{CaSiO}_{3}$ can be observed as suggested in [19]). 
investigations on SPS coatings have shown that, when the suspension is injected into the plasma flow, the liquid solvent evaporates and the remaining particles can agglomerate and are expected to melt or to remain solid, according to their specific trajectory in plasma jet. When they impinge on the substrate (or on the previously deposited particles), the well-molten particles usually result in dense and flat zones, whereas the fine particles, which are likely to re-solidify in flight, create agglomerated zones with fine and partially sintered grains [18].

In the present investigation, such agglomerated areas underwent a partial crystallisation, as suggested by the development of micrometric acicular structures; an example is given in Fig. 3b. Similar needle-like crystals have already been described in the literature and they have been associated with the growth of wollastonite, $\mathrm{CaSiO}_{3}$ [19].

The development of a rough morphology may be advantageous for bioactive coatings, since the surface asperities promote both the absorption of organic metabolites and the cell attachment [20].

The ESEM inspection of the cross sections revealed that all the samples possessed a lamellar microstructure, which is typical of thermal sprayed coatings (Fig. 4). It is worth noting that the coating-substrate interfaces were continuous and almost crack-free. Moreover the microstructural features, such as the characteristic splat dimension, were relatively fine, as a result of the small particle size of the glass powders used for the SPS deposition. The coatings' thickness varied between 31 $( \pm 7) \mu \mathrm{m}$ for BGCa4 and $40( \pm 6) \mu \mathrm{m}$ for BGCa1 and hence perfectly fitted the requirements for biomedical applications. In fact it is usually reported that a coating thickness of approximately $50 \mu \mathrm{m}$ may provide a good balance between fatigue resistance and resorption times [21].

Thanks to its relatively high crystallisation temperature [10], the BG_Ca glass experienced a very limited devitrification during the deposition process. Indeed the broad band in the $20^{\circ}-35^{\circ} 2 \theta$ range of the XRD patterns (Fig. 5) confirmed that the coatings were mainly composed of glassy phase. The partial crystallisation of the glass caused the formation of silicate-based secondary phases such as wollastonite $\left(\mathrm{CaSiO}_{3}\right)$ and $\mathrm{Ca}_{2} \mathrm{SiO}_{4}$, as proved by the weak peaks occasionally visible in the XRD patterns. The presence of wollastonite detected by the diffraction is consistent with the SEM observation of typical needle-like crystals, as mentioned before (Fig. 3b). Qualitative evaluations of the XRD patterns also suggest that the BGCa1 and BGCa2 samples possess larger amounts of crystalline phases (particularly wollastonite) than the BGCa3-BGCa5. This may be due to the high heat flux delivered from the plasma jet to the deposited material, since these samples were produced using the shortest spray distance (Table 2). This heat flux may have either slowed down the cooling of flattened splats, promoting crystallisation during splat formation, or caused re-crystallisation of previously deposited glass splats during subsequent torch scans.

It is worth noting that the development of wollastonite, which is characteristic of this $\mathrm{CaO}-$ rich glass formulation [10], is expected to

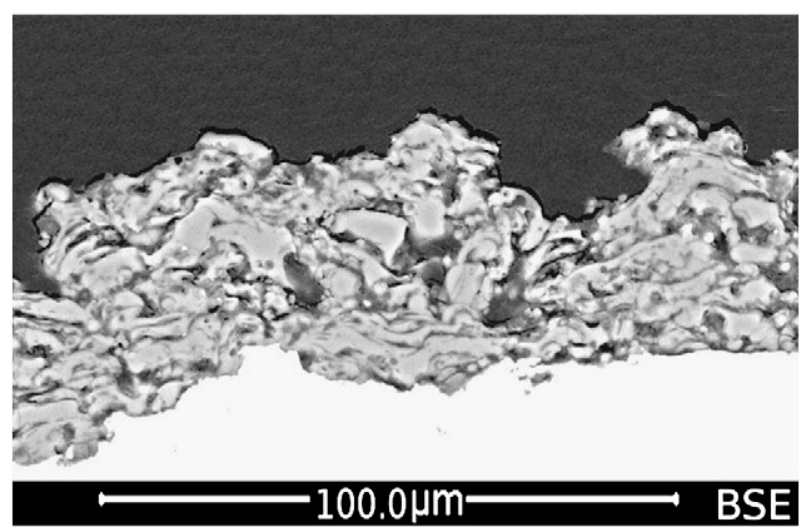

Fig. 4. SEM micrographs (secondary electrons) representative of the cross-section microstructure of the final coatings (BGCa1 run).

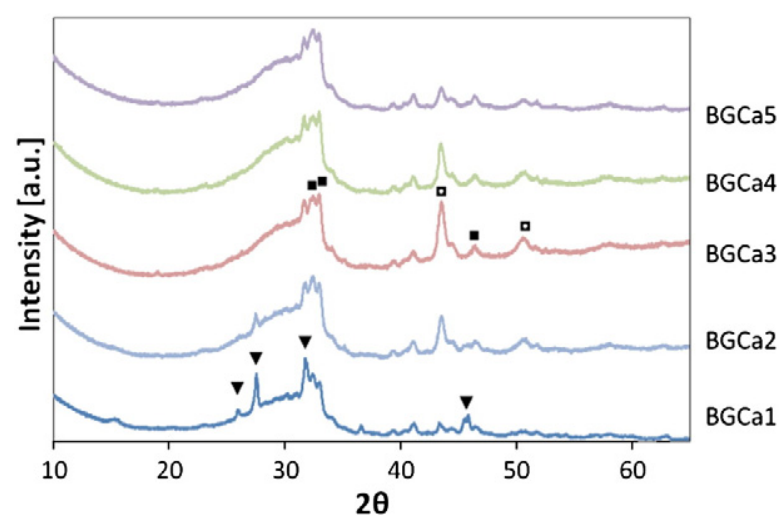

Fig. 5. XRD patterns of the as-sprayed samples. Symbols: $\mathbf{\nabla} \mathrm{CaSiO}_{3}, \mathbf{\square} \mathrm{Ca}_{2} \mathrm{SiO}_{4}$ and $\mathbf{Q}$ substrate.

preserve the bone-bonding ability of the coatings, since this material is also highly bioactive [22].

To conclude, the XRD patterns presented well-defined peaks which can be attributed to the metal substrate, due to the relatively low thickness of the coatings and the limited X-ray absorbance capacity of the glass.

\subsection{Mechanical properties of as-sprayed samples}

The Vickers hardness and the elastic modulus of the coatings were determined via depth-sensing nano-indentation. From the results reported in Table 3, it is possible to observe that the BGCa4 sample exhibited the lowest values of both Vickers hardness and elastic modulus. This is not surprising, since such coating was processed under less favourable spraying conditions (Table 2) with respect to the other final samples, being the electric power relatively low (36 kW) and, at the same time, the spray distance relatively long $(70 \mathrm{~mm})$. As a result, the maximum coating temperature remained as low as $280{ }^{\circ} \mathrm{C}$ (see Table 2). This suggests that a significant fraction of the sprayed material impinged at low temperature and that there was little chance for sintering of the previously-deposited material. It should be noted that this is also the thinnest coating on Ti6Al4V substrates (Table 3). The BGCa1 coating has the best mechanical performance, suggesting that the corresponding set of deposition parameters represents a good balance between the melting capacity of plasma jet (AHF) and the in-flight residence time of the particles. The mechanical properties of the BGCa1-BGCa5 samples are slightly worse than those reported in the literature for other bioactive glass coatings. For example, in a recent contribution by Altomare et al. dedicated to Bioglass ${ }^{\circledR} 45 \mathrm{~S} 5$ coatings obtained by the high-velocity suspension flame spray (HVSFS) technique [23], the hardness was reported to reach values as high as 290-500 HV and the elastic modulus 40-57 GPa, according to the specific deposition parameters. Nevertheless a direct comparison is not straightforward, since SPS and HVSFS are inherently different techniques, although they both employ powder suspensions. In particular, the HVSFS method usually confers a higher velocity to the sprayed particles, which is likely to promote the flattening process of the splats. This is evident in the coatings investigated by Altomare et al. [23], which possess a very compact microstructure with few spherical pores. In the present contribution, instead, the coatings produced by SPS retain a greater

Table 3

Thickness and mechanical properties of coatings (mean value \pm standard deviation).

\begin{tabular}{lllll}
\hline Run & $\begin{array}{l}\text { Thickness } \\
{[\mu \mathrm{m}]}\end{array}$ & $\begin{array}{l}\text { Hardness, } \\
\mathrm{HV}_{0.05 \mathrm{~N}}\end{array}$ & $\begin{array}{l}\text { Elastic modulus } \\
{[\mathrm{GPa}]}\end{array}$ & $\begin{array}{l}\text { Critical load } \\
{[\mathrm{N}]}\end{array}$ \\
\hline BGCa1 & $40 \pm 6$ & $98 \pm 17$ & $23.1 \pm 4.2$ & $21.1 \pm 4.4$ \\
BGCa2 & $33 \pm 7$ & $66 \pm 10$ & $20.8 \pm 3.4$ & $19.3 \pm 2.7$ \\
BGCa3 & $36 \pm 9$ & $60 \pm 13$ & $21.0 \pm 3.4$ & $18.9 \pm 0.6$ \\
BGCa4 & $31 \pm 7$ & $34 \pm 6$ & $15.6 \pm 5.6$ & $18.2 \pm 5.4$ \\
BGCa5 & $39 \pm 8$ & $85 \pm 22$ & $18.2 \pm 4.3$ & $17.9 \pm 1.9$ \\
\hline
\end{tabular}


inter-splat porosity, which directly affects the mechanical properties. However it is worth noting that a richer porosity, which results in a large reactive surface, may be advantageous, since it can aid the coating bioactivity [24]. The analogy has some limits because of the different grain sizes and compositions of the feedstock powders used, since the Bioglass ${ }^{\circledR} 45 \mathrm{~S} 5$ particles sprayed by Altomare and her colleagues were finer (1-2 $\mu \mathrm{m}$ average size) than the present ones.

Even if the elastic properties of the BGCa1-BGCa5 coatings are lower than those declared in the literature for Bioglass ${ }^{\circledR} 45 \mathrm{~S} 5$ coatings obtained via HVSFS, they resemble those of the cortical bone, whose elastic modulus is in the 7-30 GPa range $[25,26]$. This could be beneficial to limit the so-called stress shielding effect, which may cause a dangerous reduction in bone density after implantation.

The critical loads were defined by combining the analysis of the acoustic emission and the ESEM inspection of the scratch lines, as shown in Fig. 6. The results were similar for all the final coatings and they were comparable to those generally found in the literature for bioactive glass coatings, namely $16-32 \mathrm{~N}$ [4].

\subsection{In vitro tests}

As shown in Figs. 7 and 8, the ESEM investigation of the samples after soaking in SBF indicates the progressive development of the cauliflowerlike agglomerates which are characteristic for in-vitro grown HCA [27]. According to the growth rate of such dome-like features, the coatings on Ti6Al4V substrates can be classified in two groups: the samples BGCa3, BGCa4 and BGCa5, belonging to the first group (1G), showed well-defined formations already after one day of immersion in SBF (Fig. 7); the other two coatings, belonging to the second group (2G), reached well-defined spherical structures after 3 days in SBF (Fig. 8). It is likely that such difference in the coatings' reaction rate results from their microstructure; specifically, the coatings possess a different porosity, which leads to dissimilar reacting and exchanging surfaces which, in turn, are responsible for different behaviours in SBF. In fact the BGCa1 and BGCa2 coatings (belonging to $2 \mathrm{G}$ ) were processed with a lower spray distance than those belonging to $1 \mathrm{G}$, therefore they were more affected by the heat flux from the plasma jet. As a consequence, the $2 \mathrm{G}$ coatings were mainly formed by the dense zones described previously and they were less porous than the $1 \mathrm{G}$ samples. This fact delayed the reaction between the $2 \mathrm{G}$ coatings and the SBF. Moreover, the $2 \mathrm{G}$ coatings (BGCa1 and BGCa2) contained a larger amount of crystalline phases (as seen in Section 3.2 and Fig. 5), which may have reduced the rate of

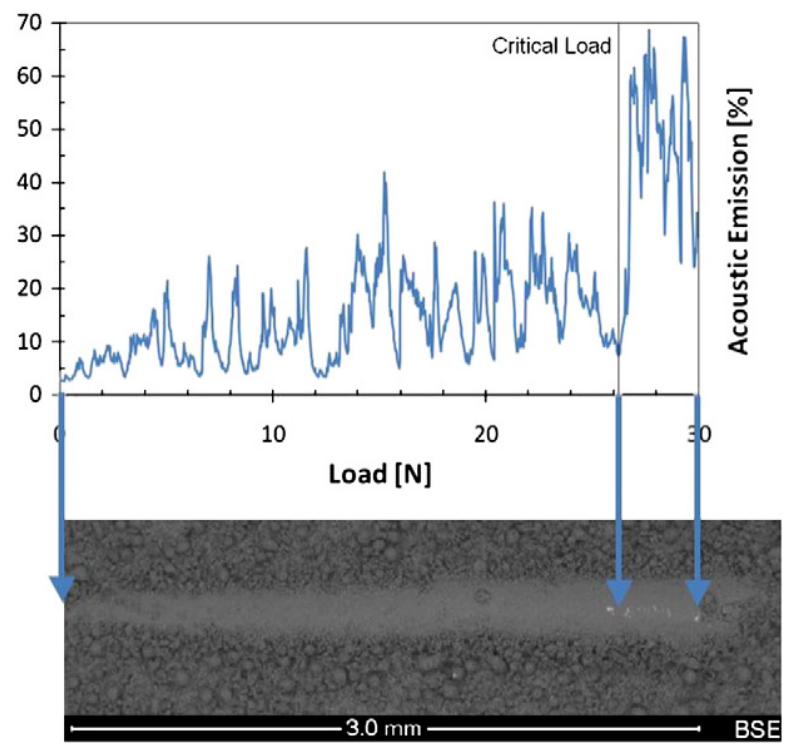

Fig. 6. Definition of the critical loads for the BGCa1 sample by combining the acoustic emission analysis and the ESEM inspection (backscattered electrons).
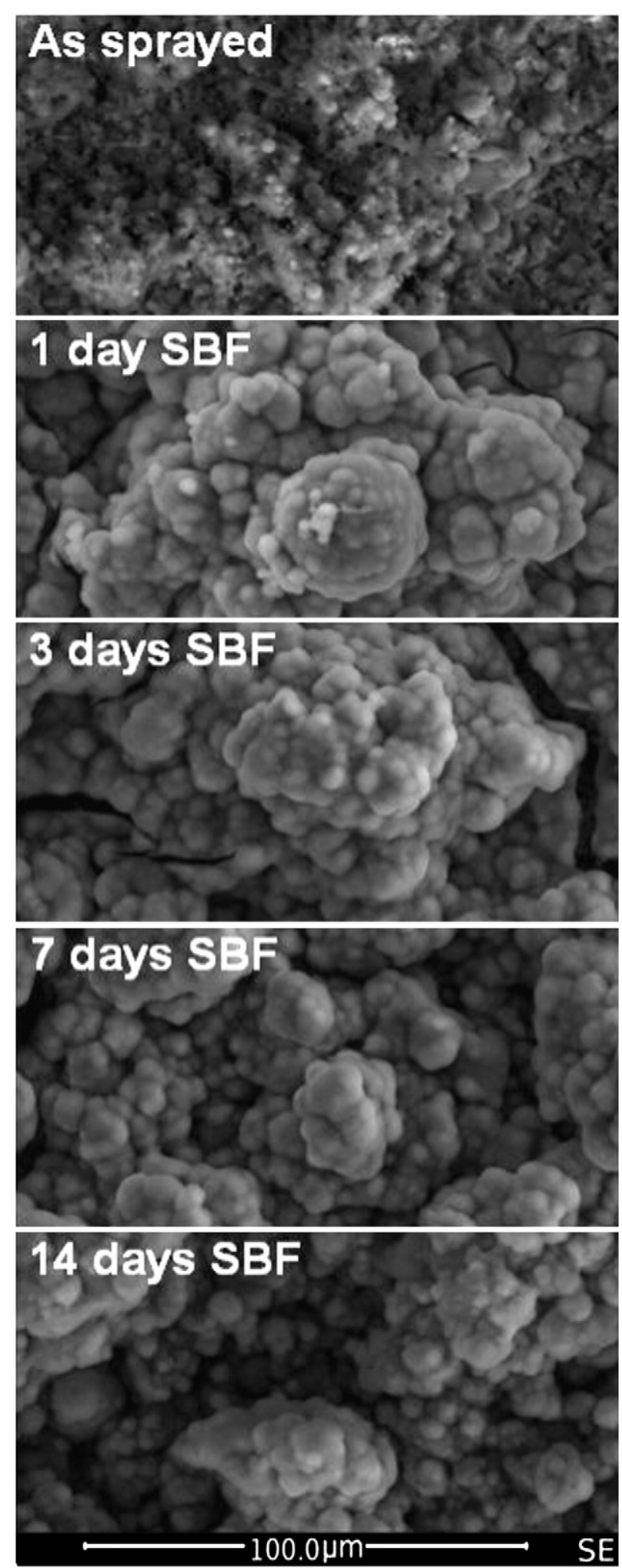

Fig. 7. Surface evolution of the samples belonging to the first group (1G) immersed in SBF for increasing times (BGCa3 run).

dissolution of the coatings since crystalline phases are typically less reactive than glassy ones.

The difference between the two groups can be appreciated also by the inspection of the cross sections. In fact, already after one day in SBF, the cross-section of a $1 \mathrm{G}$ sample typically shows a multi-layered structure, which is exemplified in Fig. 9. The X-EDS chemical analysis, supported 

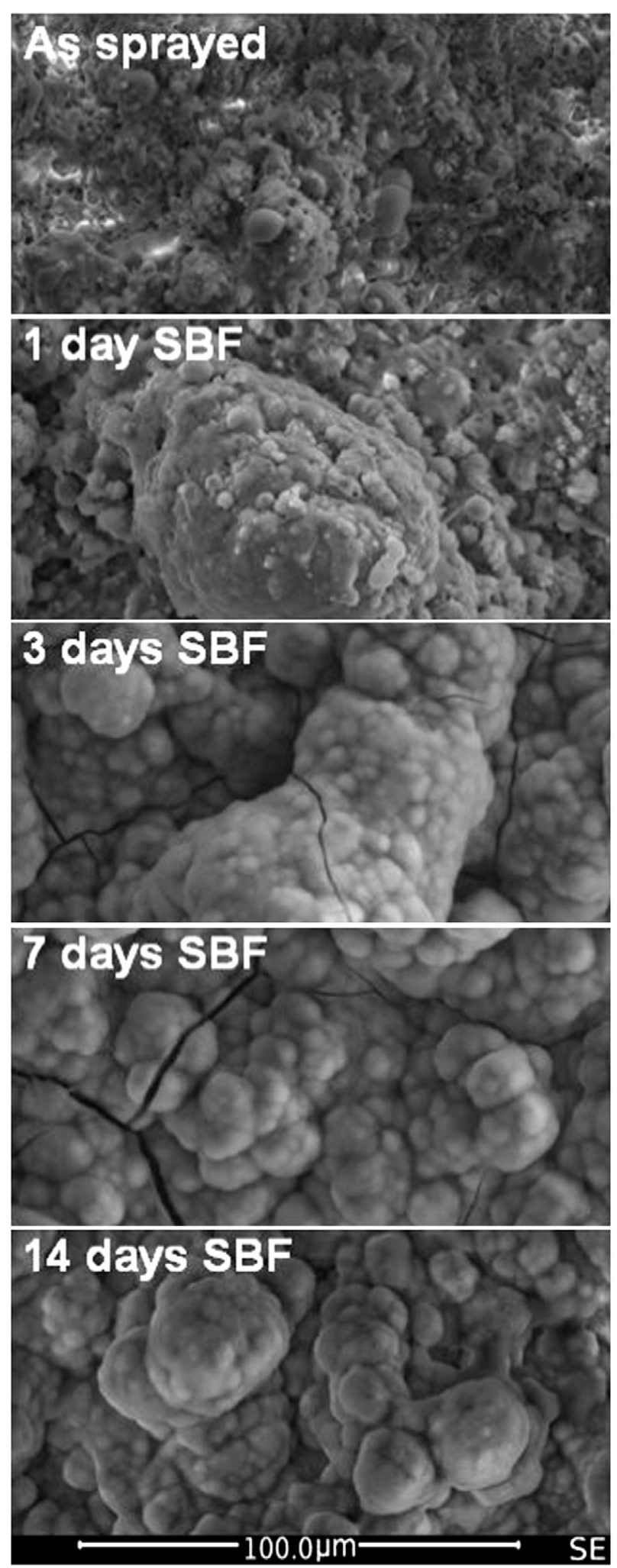

Fig. 8. Surface evolution of the samples belonging to the second group (2G) immersed in SBF for increasing times (BGCa1 run).

by the available literature [28], makes it possible to identify the strata according to the following scheme:

- superficial layer, which is rich in calcium and phosphorus (zone 1 in Fig. 9): hydroxy-carbonated-apatite;
- intermediate layer, which is mainly composed of silicon oxide (zone 2 in Fig. 9): silica gel;

- deep layer, which still preserves the composition of the starting feedstock (zone 3 in Fig. 9): residual glass splats.

The clear distinction between such layers was delayed up to the third day of immersion in SBF for the 2G samples. However, the stratification progressively disappeared with increasing soaking times and, after 14 days in SBF, all the surface deposits were entirely composed of calcium phosphate with a few residual traces of silicon. This result suggests that the original glass coatings were gradually resorbed and then completely replaced by the re-precipitated hydroxyapatite (HA). It is worth noting that, according to the ESEM and X-EDS observations, the reaction kinetics between the coatings and the SBF is (qualitatively) analogous to that commonly reported in literature for silicate-based bioactive glasses in bulk form, since a gradual growth of HA may be observed, mediated by the transient development of a silica gel layer $[3,4,11]$.

The diffraction patterns of the samples immersed in SBF (Fig. 10) are consistent with the ESEM data. The identification of the HA was based on a wide peak at about $2 \theta=32^{\circ}$, which corresponds to the main peak of hydroxyapatite, and a secondary peak at about $2 \theta=26^{\circ}$. The XRD spectra of $1 \mathrm{G}$ samples showed such peaks already after one day of immersion, whereas the spectra of the $2 \mathrm{G}$ samples exhibited them after three days of immersion. In both cases, the broad shape of the XRD peaks indicates that the hydroxyapatite had a microcrystalline and defective structure, as often stated for HA precipitated in SBF [29].

Besides, in the XRD patterns of sample $2 \mathrm{G}$, the peaks of the crystalline phases (most notably, those belonging to wollastonite) became more clearly recognisable after 1 day of soaking, which suggests that the reactivity of crystalline phases with the SBF solution is slower than that of the glassy phase. This corroborates to the previous comments on the role of crystallinity in determining the behaviour of the $1 \mathrm{G}$ and $2 \mathrm{G}$ samples. For increasingly long soaking times, the wollastonite peaks progressively got weaker and eventually disappeared. This indicates that crystalline phases also react with SBF, consistently with the mentioned reports on the bioactivity of wollastonite, although at a slightly lower rate than the glassy phase. Therefore the BG_Ca glass represents an interesting solution, since it basically remains amorphous, and hence bioactive, after the deposition and even the secondary crystalline phases resulting from the SPS process are still bioactive.

The Raman spectra, shown in Fig. 11, followed the same evolution for all samples. In fact, independently of the spraying parameters, a peak at about $970 \mathrm{~cm}^{-1}$ appeared already after one day in SBF; then, with increasing immersion times, such peak shifted towards $960 \mathrm{~cm}^{-1}$. Two secondary peaks were detectable after 3 days of soaking. To conclude, an additional peak appeared at $1070 \mathrm{~cm}^{-1}$ for longer times. All these peaks are consistent with the typical pattern of HA grown in SBF. More precisely, the Raman spectra evolution was similar to that previously observed for bulk glasses $[10,11]$. In fact, the peak at $970 \mathrm{~cm}^{-1}$, which is associated to the phosphate groups, quickly moved to $960 \mathrm{~cm}^{-1}$, which is the main peak of hydroxyapatite. Similarly, also the secondary multiple peaks were due to hydroxyapatite, while the peak at $1070 \mathrm{~cm}^{-1}$ was caused by the carbonate group, thus suggesting that the growing hydroxyapatite was carbonated, as often stated in the literature concerning SBF tests on bioactive glasses [11].

It is interesting to note that the XRD and SEM analyses allowed dividing the samples into 2 groups based on their reaction rate (Figs 7, 8 and 10). Inversely, the Raman spectra evolution was the same for all samples (Fig. 11). This indicates that, regardless of the differences in the reaction rates revealed by the ESEM and XRD studies, all the samples were immediately covered by a very thin layer of hydroxyapatite when immersed in SBF. Therefore the micro-Raman spectroscopy, which is highly sensitive to the surface of the samples, was not able to detect the differences between the coatings. 


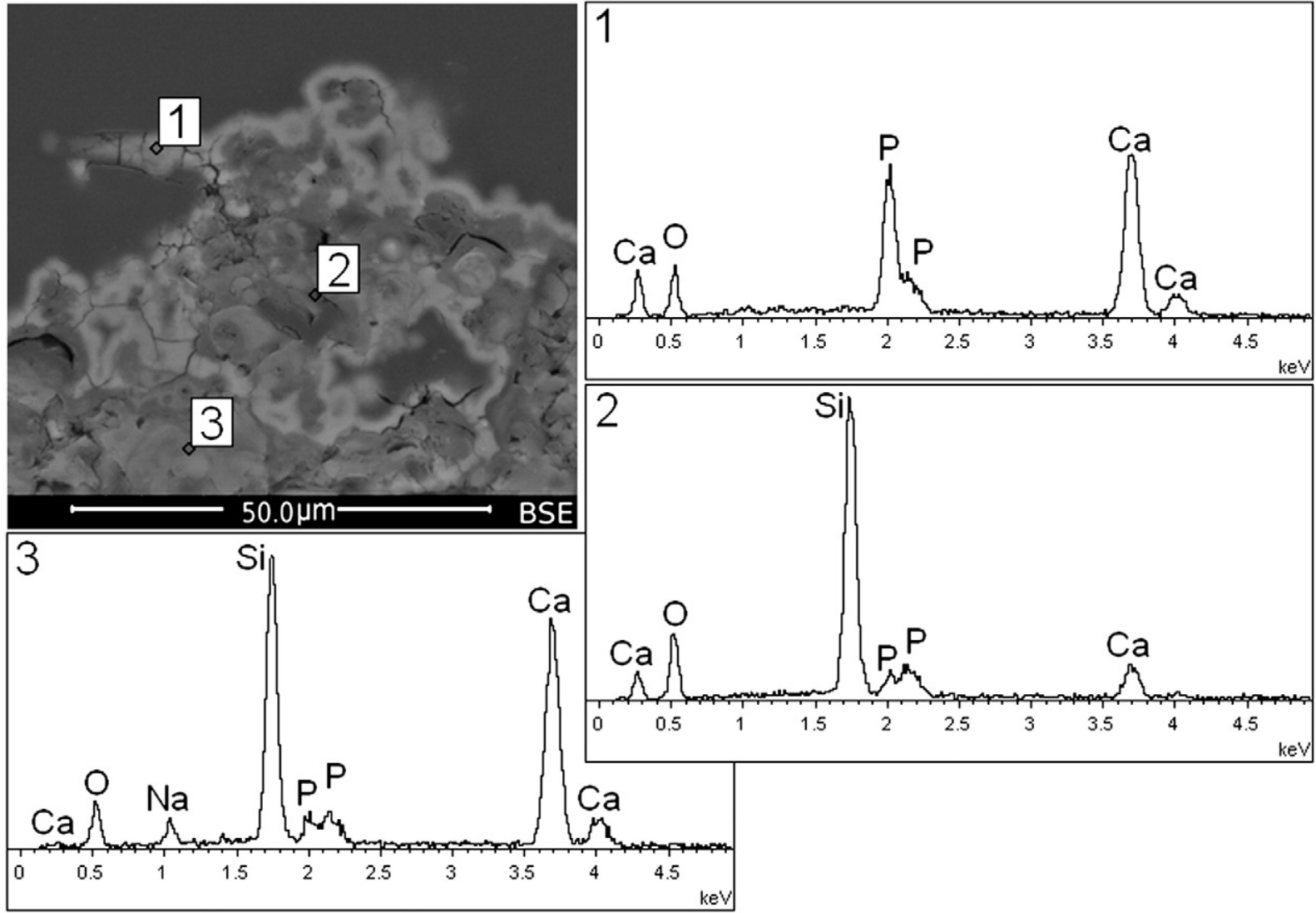

Fig. 9. ESEM micrograph (backscattered electrons) and EDS microanalysis of the cross section of a sample belonging to the first group (BGCa4) after 1 day of soaking in SBF.

\section{Conclusions}

In this study, the innovative suspension plasma spray technique was employed to obtain bioactive coatings starting from a suspension of micron-sized glass powders. A preliminary screening of processing parameters revealed that the microstructure was mainly governed by the hydrogen flow rate, the spray distance and the electric power input to plasma. Glass coatings suitable for biomedical applications were achieved working with a hydrogen flow rate of $7.5 \mathrm{slpm}$, a spray distance of 50 to $70 \mathrm{~mm}$ and a power of 36 to $40 \mathrm{~kW}$. The use of a bioactive glass, characterized by a reduced tendency to crystallise, helped to obtain amorphous coatings, with a very limited development of

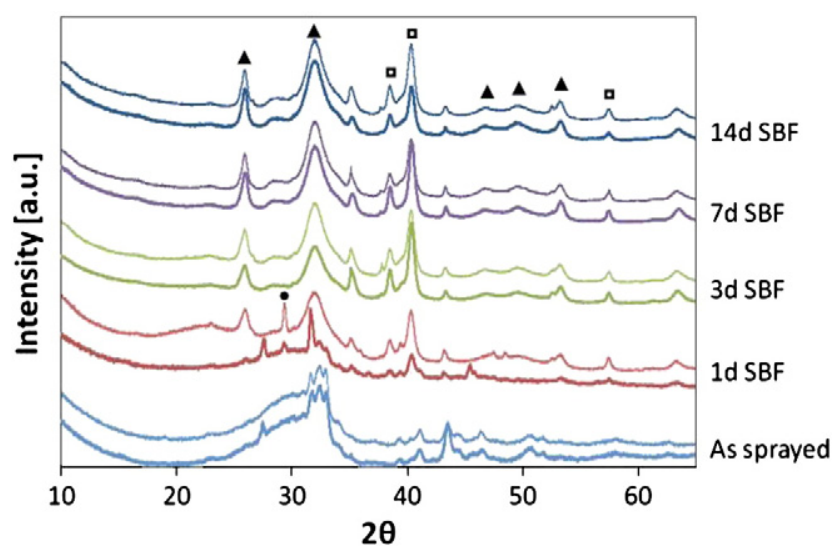

Fig. 10. XRD patterns of the samples immersed in SBF for increasing times: thin lines correspond to the sample BGCa5, representative of the first group (1G), and bold lines correspond to the sample BGCa2, representative of the second group (2G). Symbols: $\boldsymbol{\Delta}$ hydroxyapatite, $\mathbf{Q} \mathrm{Ti} 6 \mathrm{Al} 4 \mathrm{~V}$, and $\bullet \mathrm{CaCO}_{3}$. wollastonite. Both the thickness and the mechanical properties of the coatings are adequate for biomedical applications in orthopaedic implants.

The SBF tests proved that all the coatings were able to develop a surface layer of hydroxy-carbonated-apatite when immersed in a simulated body fluid, following interaction mechanisms previously observed also for bulk bioactive glasses. Nevertheless the overall reaction kinetics, especially in the first days of immersion, were influenced by the coatings' porosity and degree of crystallinity, which were primarily controlled by the spray distance.

Although this is one of the first studies conducted on bioactive glass coatings produced by SPS, the results are very encouraging;

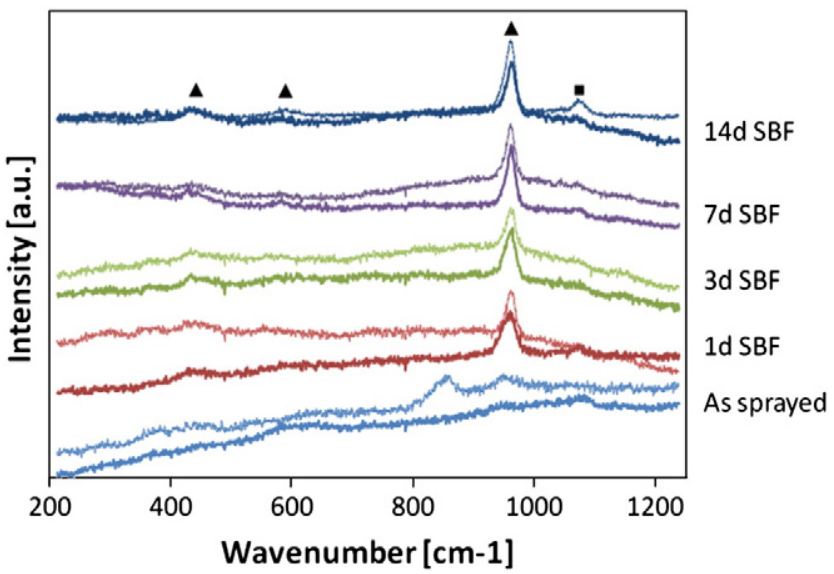

Fig. 11. Raman spectra of the samples immersed in SBF for increasing times: thin lines correspond to the sample BGCa5, representative of the first group (1G), and bold lines correspond to sample BGCa1, representative of the second group (2G). The Raman peaks correspond to the vibrations of: $\boldsymbol{\Delta}$ hydroxyapatite, and $\mathbf{\square}$ carbonate group. 
nevertheless further optimization is necessary to make the process suitable for an industrial scale-up.

\section{Acknowledgements}

The Vinci Program (Italian-French University) support for A. Cattini is gratefully acknowledged.

\section{References}

[1] L.L. Hench, J. Mater. Sci. Mater. Med. 17 (2006) 967.

[2] M.N. Rahaman, D.E. Day, B.S. Bal, Q. Fu, S.B. Jung, L.F. Bonewald, A.P. Tomsia, Acta Biomater. 7 (2011) 2355.

[3] L.L. Hench, Biomaterials 19 (1998) 1419

[4] A. Sola, D. Bellucci, V. Cannillo, A. Cattini, Surf. Eng. 27 (2011) 560.

[5] M. Geetha, A.K. Singh, R. Asokamani, A.K. Gogia, Prog. Mater. Sci. 54 (2009) 397.

[6] M.F. Morks, A. Kobayashi, Mater. Sci. Eng. B 139 (2007) 209.

[7] L. Łatka, L. Pawłowski, D. Chicot, C. Pierlot, F. Petit, Surf. Coat. Technol. 205 (2010) 954.

[8] L. Pawłowski, Surf. Coat. Technol. 203 (2009) 2807.

[9] M.W.G. Lockyer, D. Holland, R. Dupree, J. Non-Cryst. Solids 188 (1995) 207.

[10] A. Sola, D. Bellucci, M.G. Raucci, S. Zeppetelli, L. Ambrosio, V. Cannillo, J. Biomed. Mater. Res. Part A 100A (2012) 305.

[11] D. Bellucci, G. Bolelli, V. Cannillo, A. Cattini, A. Sola, Mater. Charact. 62 (2011) 1021.
[12] Y.-P. Zeng, D.-l. Jiang, J.-P. Werner, P. Greil, Mater. Lett. 57 (2002) 463

[13] W.C. Oliver, G.M. Pharr, J. Mater. Res. 6 (1992) 1564.

[14] T. Kokubo, H. Takadama, Biomaterials 27 (2006) 2907.

[15] R. Asthana, A. Kumar, N. Dahotre, in: Materials Science in Manufacturing, Academic Press, Elsevier, Burlington-San Diego-London, 2006, p. 329.

[16] L. Pawłowski, in: The Science and Engineering of Thermal Spray Coatings, Wiley, Chichester, 2008, p. 197.

[17] S. Kozerski, L. Pawłowski, R. Jaworski, F. Roudet, F. Petit, Surf. Coat. Technol. 204 (2010) 1380.

18] L. Łatka, S.B. Goryachev, S. Kozerski, L. Pawłowski, Materials 3 (2010) 3845.

[19] H. Arstila, L. Hupa, K.H. Karlsson, M. Hupa, J. Non-Cryst. Solids 354 (2008) 722.

[20] D.D. Deligianni, N.D. Katsala, P.G. Koutsoukos, Y.F. Missirlis, Biomaterials 22 (2001) 87.

[21] K.A. Gross, C.C. Berndt, Rev. Mineral. Geochem. 48 (2002) 631.

[22] X. Liu, C. Ding, P.K. Chu, Biomaterials 25 (2004) 1755.

[23] L. Altomare, D. Bellucci, G. Bolelli, B. Bonferroni, V. Cannillo, L. De Nardo, R. Gadow, A. Killinger, L. Lusvarghi, A. Sola, N. Stiegler, J. Mater. Sci. Mater. Med. 22 (2011) 1303

[24] C. Vitale-Brovarone, F. Baino, F. Tallia, C. Gervasio, E. Verné, J. Mater. Sci. Mater. Med. 23 (2012) 2369.

[25] L.L. Hench, J. Wilson, Introduction to Bioceramics, World Scientific, 1993. (Ch. 1).

[26] T. Kokubo, H.-M. Kim, M. Kawashita, Biomaterials 24 (2003) 2161.

[27] D.K. Pattanayak, Mater. Sci. Eng. C 29 (2009) 1709.

[28] J. Li, H. Liao, M. Sjöström, Biomaterials 18 (1997) 743.

[29] C. Vitale Brovarone, E. Verné, P. Appendino, J. Mater. Sci. Mater. Med. 17 (2006) 1069. 